\title{
Dosimetry and thyroid cancer: the individual dosage of radioiodine
}

\author{
Michael Lassmann, Christoph Reiners and Markus Luster ${ }^{1}$
}

Department of Nuclear Medicine, University of Würzburg, Oberdürrbacher Strasse 6, D-97080 Würzburg, Germany

${ }^{1}$ Department of Nuclear Medicine, University of Ulm, D-89081 Ulm, Germany

(Correspondence should be addressed to M Lassmann; Email: lassmann_m @ klinik.uni-wuerzburg.de)

\begin{abstract}
Adjuvant therapy of differentiated thyroid cancer with radioactive iodine $\left({ }^{131} I\right)$ is a standard procedure for the ablation of remnant thyroid tissue following surgery and for the treatment of iodine-avid metastases. Presently, there are two dosimetric concepts for the treatment of thyroid cancer using radioiodine: a) the bone marrow dose limited approach and b) lesion-based dosimetry. Both concepts and their clinical applications are described. In addition, the use of ${ }^{124}$ I as a diagnostic and dosimetric agent is discussed. Treatment of children and adolescents with radioiodine requires special precautions; individualized approaches in this setting are reviewed. The limitations of treatments aiming at high absorbed doses are addressed as well as the doses to normal organs. Finally, new concepts for further elaborating the potential of thyroid cancer treatment using ${ }^{131} \mathrm{I}$ are introduced.
\end{abstract}

Endocrine-Related Cancer (2010) 17 R161-R172

\section{Introduction}

Treatment of differentiated thyroid cancer (DTC) with radioactive iodine $\left({ }^{131} \mathrm{I}\right.$; often referred to as 'radioiodine') is a standard procedure for the ablation of remnant thyroid tissue following surgery and for the treatment of iodine-avid metastases. An excellent overview of the procedures, and risks related to DTC therapy with radioiodine can be found in the textbook by Schlumberger \& Pacini (2003).

The activity to be used for radioiodine therapy still remains subject to discussion. Usually, patients are treated with standard activities reflecting the physician's rating of the highest safe or 'adequate' dosage rather than with an optimized treatment activity based on prior measurement of the patient's individual biokinetics. Such a standard activity poses a risk of either underdosing the patient or of exceeding common safety limits (Van Nostrand et al. 2002, Tuttle et al. 2006).

There is a broad range of fixed activities of ${ }^{131} \mathrm{I}$ recommended to be administered (see e.g. Meier et al. (2002) and Luster et al. (2008)). In many cases, an activity between 1.1 and $3.7 \mathrm{GBq}$ is prescribed for the first radioiodine therapy after thyroidectomy in newly diagnosed DTC to ablate the remaining glandular tissue. Higher amounts of ${ }^{131} \mathrm{I}$ are given in subsequent therapies or in case of metastatic disease. Normally, the activity is limited for safety reasons to around 7.4 GBq. A summary on the use of fixed activities for the treatment of DTC until 2002 can be found in a review article by Menzel et al. (1996), Van Nostrand et al. (2002) and Haq et al. (2004) adopt a more aggressive approach for advanced stages of the disease employing empiric fixed activities of radioiodine of $9 \mathrm{GBq}$ (Haq et al. 2004) and $11.1 \mathrm{GBq}$ at 3-month intervals (Menzel et al. 1996).

The main disadvantage in using a fixed activity approach is the failure to consider the individuality of the patient. The 'optimal' activity of radioiodine to treat metastatic thyroid carcinoma is the lowest possible amount of radioiodine that delivers a lethal dose of radiation to the entire lesion/metastasis while minimizing side effects. Empiric fixed activities by their very nature make no attempt to determine either the minimal radioiodine activity that will deliver a lethal dose or the maximum allowable reasonably safe absorbed dose.

An additional shortcoming is that multiple empiric fixed activities may not be equivalent to the same total radioiodine absorbed dose calculated by dosimetry given at one time. Dose rate is crucial, and thus, multiple smaller doses may have lower therapeutic benefit than the same total dose given at one time 
(see e.g. Samuel et al. (1998)). On top of that, previous nonlethal doses may reduce the effectiveness of subsequent doses (Medvedec 2005).

Therefore, the rationale for using a dosimetry-based approach is to replace the conventional fixed activity regimen by a modern setting, which allows the administered therapeutic activity to be increased while avoiding undesired side effects. Using this strategy, the absorbed dose to iodine-avid tissue (remnants/metastases) can be optimized without inducing potential toxicity.

One could also consider decreasing the administered activity based upon a dosimetric approach as proposed by Hänscheid et al. (2009). However, for establishing such an approach, clinical data are missing at present.

As has been pointed out in a recent article on radioiodine therapy dosimetry by Salvatori \& Luster (2010), it is to be expected and hoped that in the near future, radionuclide treatments with administered activities based on prescribed absorbed doses, as used in external beam radiation therapy, will become commonplace, improving the efficacy of the treatment and, therefore, individual clinical outcomes.

\section{Dosimetric concepts}

Presently, there are two dosimetric concepts for the treatment of thyroid cancer using radioiodine:

a) The bone marrow dose limited approach, originally described by Benua et al. (1962):

This concept is mainly aiming at the safety of the treatment. Blood, as a surrogate of the organ at risk, i.e. the red bone marrow, was considered as the critical organ in this approach. The generally accepted surrogate dose threshold to avoid serious myelotoxicity is a blood absorbed dose of 2 Gy.

b) The lesion-based dosimetry, based mainly on the data of Maxon et al. (1983) and Maxon \& Smith (1990):

This concept aims at improving the efficacy of the treatment by achieving an absorbed dose threshold of more than $300 \mathrm{~Gy}$ to remnants and of more than $80 \mathrm{~Gy}$ to iodine-avid tissue. The value of $80 \mathrm{~Gy}$ was originally defined for the treatment of cervical lymph node metastases, and is assumed to be accurate also for distant metastases.

\section{Bone marrow dose limited approach}

The method originally reported by Benua et al. (1962) and, applied to a larger patient series, by Leeper (1985) allows an estimate to be calculated for the radiation dose that will be delivered to the hematopoietic system from each $\mathrm{GBq}$ administered to any patient. This is possible because it involves the collection of data over the course of four or more days after the administration of a tracer activity of ${ }^{131} \mathrm{I}$ to the patient. The measurement procedures involve serial blood sampling and serial measurements of the patient's whole body activity using a calibrated probe.

Given the time period in which this methodology was developed, it follows that the dosimetry calculations were based on the classic formulations rather than on the Medical Internal Radiation Dose Committee of the Society of Nuclear Medicine (MIRD) absorbed fraction method (Schlumberger \& Pacini 2003, Lassmann et al. 2008). Generally, both approaches are in good agreement; however, the patient specificity is improved using the MIRD formalism as described by the Lassmann et al. (2008). Furthermore, it should be emphasized that these calculations yield the radiation dose to the wholeblood compartment and not to the bone marrow directly. In the original setting of Benua, a subgroup of patients, namely those that received 2 Gy or less to the blood, showed no serious side effects.

In the classic Benua approach, the blood is considered as the critical organ that is irradiated either from the particles emitted from activity in the blood itself or from the emissions originating from activity dispersed throughout the remainder of the body.

The maximum treatment activity $A_{\text {administered }}$ is calculated as the activity of ${ }^{131} \mathrm{I}$ that would deliver a combined beta particle absorbed dose $D_{\beta}$ and gamma ray absorbed dose $D_{\gamma}$ of 2 Gy to the blood compartment, and is given by:

$$
\mathrm{A}_{\text {administered }}(\mathrm{MBq})=\frac{2 \mathrm{~Gy}}{\mathrm{D}_{\beta}(\mathrm{Gy} / \mathrm{MBq})+\mathrm{D}_{\gamma}(\mathrm{Gy} / \mathrm{MBq})}
$$

$D_{\beta}$ which is calculated by integrating the blood activity biokinetics (measuring blood samples in a gamma-counter), whereas the $D_{\gamma}$-dose is taken from serial measurements of the patient with a gamma-probe.

Individual patient-specific geometry parameters such as weight and height are not considered when assessing the activity to be administered. In addition, the measurements are often not repeated during therapy in order to compare the estimated pretherapeutic absorbed dose to the absorbed dose achieved after therapy.

Recently, in the framework of several international multicenter trials for the study of the biokinetics after the use of recombinant human TSH (rhTSH; Luster et al. 2003, Hänscheid et al. 2006), the absorbed dose 
to the blood was calculated with a modified method deduced from a procedure originally described by Thomas et al. (1993). Refinements to this model have been introduced to account for:

1) the contribution from penetrating radiation from activity in distant blood to the blood dose,

2) the mass dependency of the $S$ value representing the radiation from the total body to the blood, and

3) a mean value $S_{\text {blood } \leftarrow \text { blood }}$ representing an average for blood circulating in vessels of different diameters with different $S$ values (Lassmann et al. 2004).

The equation for the mean absorbed dose $\overline{\mathrm{D}}_{\text {blood }}$ to the blood per unit administered activity becomes:

$$
\begin{aligned}
\frac{\overline{\mathrm{D}}_{\text {blood }}}{\mathrm{A}_{0}}\left(\frac{\mathrm{Gy}}{\mathrm{GBq}}\right)= & 108 \times \tau_{\mathrm{ml} \text { of blood }}(\mathrm{h}) \\
& +\frac{0.0188}{(\mathrm{wt}(\mathrm{kg}))^{2 / 3}} \times \tau_{\text {total body }}(\mathrm{h})
\end{aligned}
$$

$\tau_{\text {source }}$ stands for the residence time in a source organ representing the integral of the activity-time curve in the source organ (cumulated activity) divided by the administered activity; wt represents the patient's weight in $\mathrm{kg}$. The modified expression accounts for the activity in distant blood vessels and the influence of the individual patient mass.

Therefore, only two compartments need to be monitored for radioactivity: blood and the whole body. The activity in the blood is determined from periodic heparinised blood samples. The activity in the whole body, i.e. remaining in the patient, could be monitored redundantly using independent techniques: $24 \mathrm{~h}$ urine collections, whole-body counting with a probe using a fixed geometry and, if applicable, conjugate views of whole body scan obtained by a dual-headed gamma camera.

Details of the recommended data sampling frequency and of the calculation process can be found in the recently published standard operational procedures (SOP) of the EANM (Lassmann et al. 2008). In Fig. 1, an example of a post-therapeutic dose assessment after administration of $3.6 \mathrm{GBq}{ }^{131} \mathrm{I}$ is given. Panel A shows the measured data and the corresponding fits (whole-body dose rate and blood activity normalized to the administered activity), and panel $\mathrm{B}$ shows the temporal change of the absorbed dose and the dose rate.

The presented model might not to be applied in the presence of extensive metastatic bone involvement, as
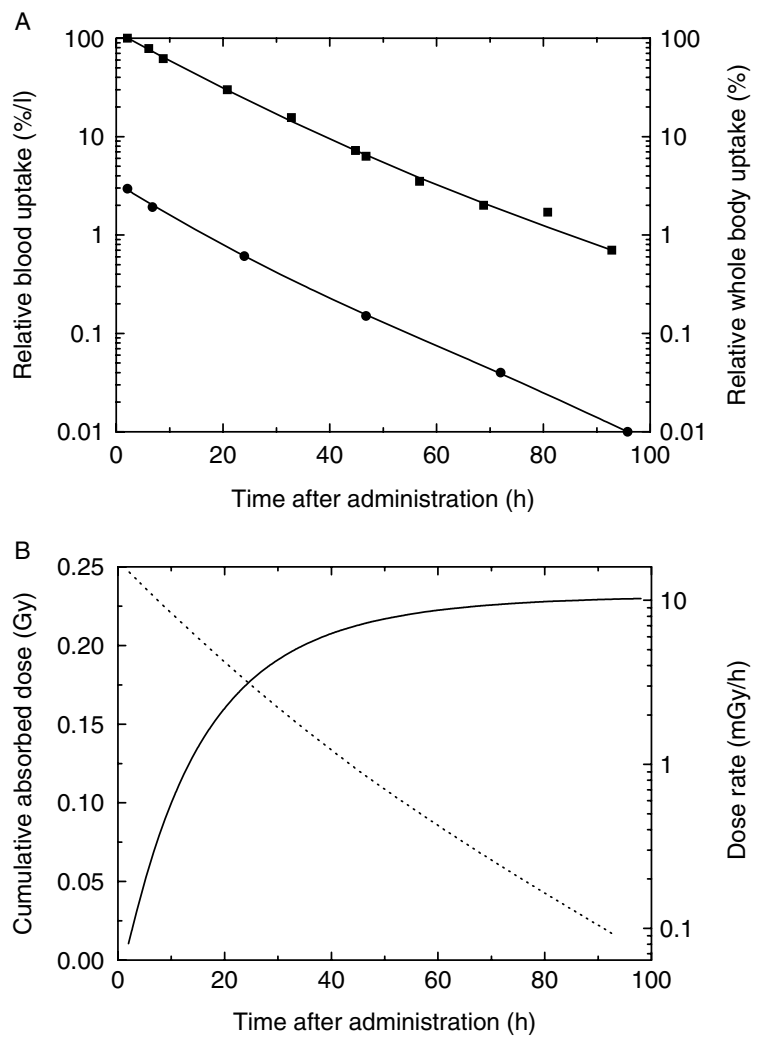

Figure 1 Time curves of a post-therapeutic blood dose assessment according to the EANM SOP (Lassmann et al. 2008) after administration of $3.6 \mathrm{GBq}^{131} \mathrm{I}$ (absorbed dose to the blood: $0.23 \mathrm{~Gy}$ ). (A) Measured relative uptake and corresponding bi-exponential fits per liter of blood (filled circles, left axis) and of the whole body (filled squares, right axis).

(B) Cumulative absorbed dose to the blood (solid line, left axis) and absorbed dose rate (dotted line, right axis).

the blood-based absorbed dose calculation could underestimate the absorbed dose to the red marrow.

It should be carefully applied in patients affected by diffuse pulmonary micro-metastases, since the critical organ could be the lung itself instead of the hematopoietic red marrow. In the Benua approach, fatal complications were avoided after restricting the incorporated activity at $48 \mathrm{~h}$ to $4.5 \mathrm{GBq}$ and, in the case of diffuse lung metastases, to $<3 \mathrm{GBq}$.

Sgouros et al. (2006) used the 3-GBq activity retention limit originally introduced by Benua et al. (1962) in order to derive patient-specific lung absorbed doses and dose rates in order to account for differences in lung geometry. The authors obtain a dose-rate constraint value, which yields when applied to an adult male phantom and to the phantom of a 15 -year-old yields 48-h activity limits of 3.72 and $2.45 \mathrm{GBq}$ respectively. Using this approach, severe 
radiation-induced lung toxicity, expected at normal lung absorbed doses of 25-27 Gy, is avoided.

The strengths of the blood-based dosimetry approach are (Lassmann et al. 2008) as follows:

1) determination of the maximal safe activity of radioiodine for each patient individually;

2) identification of patients for whom empiric fixed activities are not safe;

3) the potential to administer higher activities at once instead of multiple administrations of lower activities in a 'fractionated' therapy in order to avoid changes in tumor/lesion biokinetics after multiple therapies that have been observed, e.g. by Samuel et al. (1998);

4) no necessity to determine lesion volumes;

5) a long history of use in several institutions;

6) an expected increase in the probability of curing patients in and advanced stages of the disease with fewer courses of therapy.

Limitations that need to be mentioned are as follows:

1) a benefit of the strategy is plausible but no valid clinical data exist on improved response and/or outcome rates;

2) the absorbed dose to the tumor is not known. Higher activities might be administered without achieving a higher therapeutic effect when using this methodology;

3) the current debate regarding the issue of 'stunning' argues that diagnostic administrations of ${ }^{131}$ I could alter tumor/lesion biokinetics and, consequently, the absorbed dose in a subsequent treatment.

\section{Lesion-based dosimetry}

The objective of remnant or lesion dosimetry is to determine the radioiodine activity that delivers the recommended absorbed dose of radiation to ablate thyroid remnant or to treat metastatic disease while minimizing the risk to the patient. The absorbed doses are traditionally considered to be 300 and $80 \mathrm{~Gy}$ respectively (Maxon et al. 1983, Maxon \& Smith 1990). In contrast to these pretherapeutic dosimetric procedures, the radiation absorbed dose delivered by the ${ }^{131}$ I therapeutic activity itself to the thyroid remnant or to lesions also may be measured post-therapy, attempting to correlate this dose with clinical results.

In order to perform these calculations, it is necessary to measure the uptake and clearance of ${ }^{131} \mathrm{I}$ from identifiable thyroid remnants and/or metastatic lesions. This calculation of lesion dose is generally based on the same model as described above. For example, if the lesions become smaller, the nodule module of OLINDA/EXM could be used for a spherical model of the tumor and/or the lesion (Stabin et al. 2005). Furthermore, if the dimensions of the lesions are smaller than $\sim 5 \mathrm{~mm}$ (assuming that this could be accurately determined), then the range of the beta-particles can no longer be neglected in the dose calculation (Li et al. 2001).

In order to determine the concentration of ${ }^{131} \mathrm{I}$, one needs to know how much activity is contained in the lesion. One way in which this can be examined is based on an analysis of selected regions of interest on conjugate view gamma-camera images (Siegel et al. 1999). These images are obtained at several time points measured from the time of administration of a tracer activity. Typically, subsequent image acquisition would happen up to $96 \mathrm{~h}$, but later samples might be necessary if the uptake and clearance are delayed. In addition, transmission images to correct for attenuation in the region of the lesion, as well as images of a standard for calibration purposes, might be necessary (Siegel et al. 1999). A curve-fitting procedure is then used to evaluate the assumed single-exponential halflife value and to extrapolate the curve to zero time in order to determine the initial activity in the lesion.

Particularly when using probe data or planar scintigrams, another important parameter needed for calculating the specific activity is the mass of the lesion. For ablation therapies, the various procedures for determining the remnant size such as ultrasound or computed tomography (CT) are unreliable as no validated method exists to exactly determine the mass of thyroid remnants shortly after surgery (Hänscheid et al. 2006); consequently, one has to be careful when reporting absorbed doses to the target tissue. For lesion dosimetry, higher spatial resolution images like CT can be used for attenuation correction and to determine the mass.

The main disadvantages of a lesion-based approach to thyroid cancer treatment dosimetry are (Lassmann et al. 2008) as follows:

1) a wide range of absorbed doses to lesions/metastases even within one patient;

2) inhomogeneous absorbed dose distributions within one lesion may result in incomplete tumor destruction;

3) a mono-exponential model may not accurately reflect the kinetics of the radioiodine in the lesion; 
4) ill-defined corrections have to be applied for the initial phase of increasing uptake (up to $\sim 24 \mathrm{~h}$ ) caused, e.g. by the delayed resorption of orally administered ${ }^{131} \mathrm{I}$;

5) an accurate measurement of the lesion mass is not always possible;

6) statistical errors in the measurements due to bad counting statistics particularly at late time points;

7) unknown biologic effectiveness of the treatment due to the potentially heterogeneous iodine uptake on a microscopic scale which cannot be accounted for in conventional scintigraphic images.

\section{Clinical findings}

\section{Bone marrow dose limited approach}

The blood or red marrow dosimetry offers not only the possibility of increasing the administered activity. The same method allows reducing the activity in order to guarantee safety of the treatment. It is a fact that the fixed activity approach, being completely blind, can give blood absorbed doses higher than $2 \mathrm{~Gy}$.

Kulkarni et al. (2006) performed pretreatment blood dosimetric studies on 127 patients with $74 \mathrm{MBq}$ of ${ }^{131} \mathrm{I}$. Then, once measured the blood-specific absorbed dose (Gy/GBq) of their patient group, they calculated the percentage of patients who would have received more than $2 \mathrm{~Gy}$ to the blood according to different hypothetical fixed activity administrations. For example, for an assumed empiric dosage of ${ }^{131} \mathrm{I}$ of $7.4 \mathrm{GBq}$, the percentage of treatments for which patients would have exceeded the 2 Gy limit were $<11 \%$. A more detailed analysis of these percentages as a function of the administered activity is shown in Fig. 2.

Tuttle et al. (2006) analyzed retrospectively 535 hypothyroid blood dosimetries in 328 patients with apparently normal renal function. They found that the maximum tolerated activity (corresponding to $2 \mathrm{~Gy}$ to blood) was $<7.4 \mathrm{GBq}$ in $8 \%$ and $<9.25 \mathrm{GBq}$ in $19 \%$ of their patients. Factors associated with a lowering of the maximum tolerated activity to $<9.25 \mathrm{GBq}$ were age at dosimetry $>45$ years, the female sex, subtotal thyroidectomy, and radioiodine-avid diffuse bilateral pulmonary metastases. Administered activities of 7.4-9.25 GBq frequently exceeded the calculated maximum tolerated activity in patients 70 years old or above. Therefore, the authors conclude that dosimetry-guided radioiodine therapy may be preferable to fixed-dose treatment strategies in older patients with thyroid cancer and in patients with

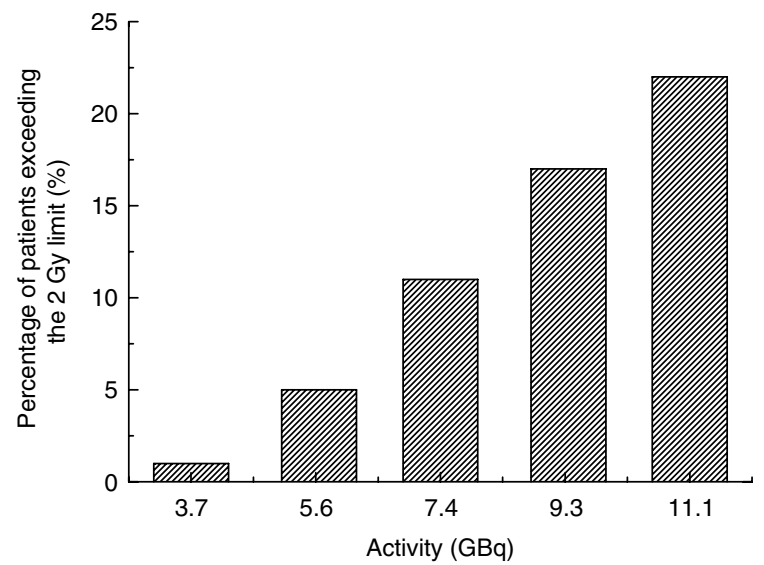

Figure 2 Percentage of patients who would have received a blood absorbed dose $>2$ Gy in case of empiric fixed activity administration (adapted from Kulkarni et al. (2006)).

radioiodine-avid diffuse bilateral pulmonary metastases, even when renal function is normal.

Recently, rhTSH was approved as an alternative to thyroid hormone withdrawal (THW) to elevate TSH for thyroid remnant ablation in DTC patients (Pacini et al. 2006). However, improved renal function causes $\sim 30 \%$ faster radioiodine clearance under euthyroidism versus hypothyroidism, and high ablation success rates are reported with diverse rhTSH-aided ${ }^{131} \mathrm{I}$ activities (Hänscheid et al. 2006, Remy et al. 2008).

An analysis of the distribution of the relative absorbed dose coefficients (the absorbed dose coefficient is defined as the absorbed dose divided by the administered activity) in 66 patients after radioiodine therapy of DTC is shown in Fig. 3 (Lassmann et al. 2007). The data are normalized to the lowest specific absorbed dose. Patients were either hypothyroid $(n=32)$ or euthyroid $(n=34)$. The mean absorbed dose coefficients for euthyroid patients are $\sim 30 \%$ lower as compared with patients after thyroid hormone withdrawal (hypothyroid). The highest absorbed dose coefficient is almost sixfold higher as compared with the data of the patient with the lowest absorbed dose coefficient. This figure illustrates a) the wide distribution of absorbed doses to the blood when using standard activities and b) the faster radioiodine clearance for euthyroid patients as compared with hypothyroid patients.

In a recent publication by Hänscheid et al. (2009), the authors discuss if a lower targeted blood absorbed dose seems reasonable for remnant ablation therapy in low risk patients. Based on formulas and assumptions presented in this work, and data derived 


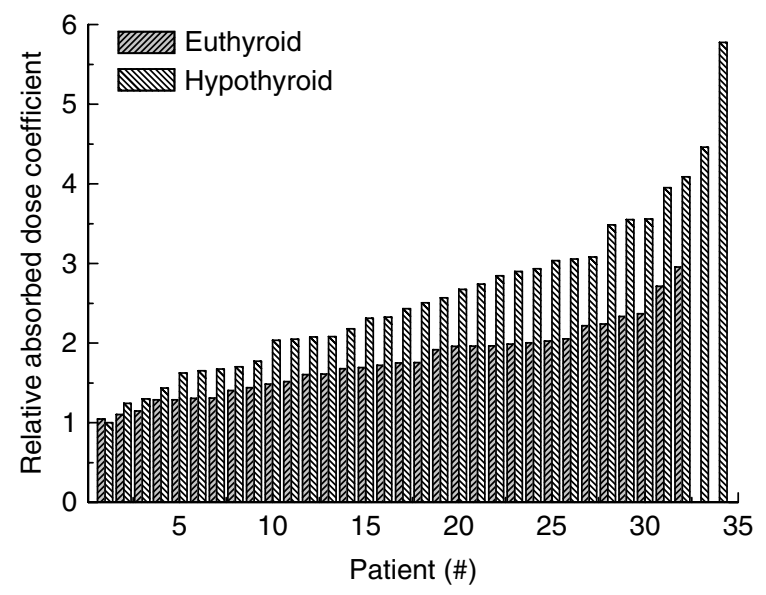

Figure 3 Relative distribution of the absorbed dose coefficients (absorbed dose to blood) after radioiodine therapy in 66 patients after thyroid hormone withdrawal (hypothyroid) or after the use of recombinant human TSH (euthyroid) (Lassmann et al. 2007). The data are normalized to the minimal absorbed dose coefficient.

from a series of patients treated with $3.7 \mathrm{GBq}{ }^{131} \mathrm{I}$ (Hänscheid et al. 2006), the mean absorbed doses to the blood were 0.45 and $0.29 \mathrm{~Gy}$ when prepared for therapy with hypothyroidism or euthyroidism using rhTSH respectively. As the rate of remnant ablation between the two groups was similar, it may be adequate to routinely select a remnant ablative activity that delivers 0.3 Gy to the blood for patients of similar disease stage. However, as blood dose-based remnant ablation therapy is not yet validated, the paper stimulates the discussion if dosimetry-based ablation rate studies should be performed.

In the case of a pediatric patient presenting with diffuse lung metastases, Song et al. (2006) demonstrated the usefulness of dose planning using a) the conventional Benua approach, b) the dose-rate constraint approach introduced by Sgouros et al. (2006) described above, and c) a Monte Carlo calculationbased approach. Directly applying the Benua method, an administered activity of $3.9 \mathrm{GBq}$ was obtained, the dose-rate constraint approach resulted in $2.4 \mathrm{GBq}$, whereas in the Monte Carlo approach the authors demonstrated that an administered activity of $1.7 \mathrm{GBq}$ resulted in a lung dose of $27 \mathrm{~Gy}$. Such discrepancy highlights the necessity of more studies to correlate between the Benua dosing method and absorbed doses to normal organs and tumors.

\section{Lesion-based dosimetry}

The objective of remnant or lesion dosimetry is to determine the radioiodine activity that delivers the recommended absorbed dose of radiation to ablate thyroid remnant or to treat metastatic disease while minimizing the risk to the patient. The doses are traditionally considered to be 300 and 80 Gy respectively (Maxon et al. 1983, Maxon \& Smith 1990). In contrast to these pretherapeutic dosimetric procedures, the radiation absorbed dose delivered by the ${ }^{131} \mathrm{I}$ therapeutic activity itself to the thyroid remnant or to lesions also may be measured post-therapy to correlate this dose with clinical results.

de Keizer et al. (2003) evaluated the tumor dosimetry following ${ }^{131}$ I treatment after rhTSH stimulation. They performed dosimetric calculations after ${ }^{131} \mathrm{I}$ tumor uptake measurements from posttreatment ${ }^{131}$ I scintigrams and tumor volume estimations from radiological images. Their reported median tumor radiation absorbed doses were between 1.3 and $368 \mathrm{~Gy}$, thus showing a high variability in the lesion absorbed doses. Only 5 out of 25 lesions investigated received a dose of more than $80 \mathrm{~Gy}$.

Similar findings were reported by Sgouros et al. (2004) who used ${ }^{124}$ I for a voxel-based pretherapeutic lesion dose assessment.

A successive change in the biokinetics of pulmonary metastases after several therapies has been observed by Samuel et al. (1998). In their study, they demonstrated that dosimetric parameters such as radioiodine uptake as a percentage of therapeutic activity, effective halflife and radiation dose delivered to the lungs which were evaluated with each therapy, showed a progressive decline in each of these parameters with successive therapies.

Chiesa et al. (2009) described a total of 17/20 lesions with doses below $80 \mathrm{~Gy}$ in their patient group. Their data also suggest that the repetition of treatment of a lesion drastically reduces its uptake, with a loss of therapeutic efficacy along the sequence of fixed activity administrations.

Some groups (Freudenberg et al. 2007, Flux et al. 2010) combine the lesion- and blood-based dosimetric approaches; however, larger patient series are warranted to further support this strategy (De Klerk \& Oyen 2009).

\section{The use of ${ }^{124}$ I for dosimetry}

Traditional radiopharmaceuticals that accurately quantify radioiodine uptake in thyroid remnants and metastases such as ${ }^{123} \mathrm{I}$ or ${ }^{131} \mathrm{I}$ have different limitations: the former presents problems regarding supply and short half-life, the latter a relatively low sensitivity and the risk of induction of the 'stunning' 
phenomenon. The recent availability of a positron emission tomography (PET) tracer such as ${ }^{124} \mathrm{I}$ has created new perspectives for PET/CT, both as an effective staging tool to detect recurrent/residual disease and for dosimetry of (metastatic) lesions.

The use of ${ }^{124} \mathrm{I}$ was proposed for quantifying volume, in vivo tumor concentration, and biodistribution for DTC (Pentlow et al. 1996, Eschmann et al. 2002, Sgouros et al. 2004). Owing to the complex decay process of ${ }^{124} \mathrm{I}$, tumor-associated quantification cannot be performed in the same way as for F-18. Pentlow et al. (1996) measured resolution, linearity, and the ability to quantify the activity contents of imaged spheres of different sizes and activities for different background activities. It was shown that the quantification process for ${ }^{124} \mathrm{I}$ could reliably reproduce the activities administered. PET with ${ }^{124} \mathrm{I}$ was also successfully applied to the measurement of thyroid volume (Crawford et al. 1997, Eschmann et al. 2002).

The first rigorous approach to PET ${ }^{124}$ I-based thyroid cancer dosimetry has been described in a publication by Sgouros et al. (2004), in which the authors showed by using the PET results as input to a fully $3 \mathrm{D}$ dose planning program spatial distributions of absorbed doses, isodose contours, dose-volume histograms, and mean absorbed dose estimates for a total of 56 tumors. The mean absorbed dose values for individual tumors ranged from 1.2 to 540 Gy. The absorbed dose distribution within individual lesions was widely distributed ranging from a minimum of $0.3 \mathrm{~Gy}$ to a maximum of 4000 Gy showing the high variability of dose ranges even within one patient.

The aims of the study by Capoccetti et al. (2009) were to evaluate the usefulness of ${ }^{124}$ I PET/CT as a tool for DTC staging immediately before ${ }^{131} \mathrm{I}$ therapy, optimizing the activity for remnant ablation, and individualizing dosimetry in patients with multiple distant metastases. In two cases, individualized pretherapeutic ${ }^{124}$ I-based dosimetry led to a maximum safe activity of 13 and $9 \mathrm{GBq}$ respectively. In 16/21 lesions studied, the absorbed was $<80 \mathrm{~Gy}$. On the basis of their findings, the authors conclude that ${ }^{124} \mathrm{I}$ PET/CT could be routinely employed in order to obtain a reliable dosimetry in patients with multiple metastases or to stimulate consideration of alternative therapies, and thereby finally improving the management of those patients; however, its use is restricted by the limited availability.

Freudenberg et al. $(2007,2009)$ performed through ${ }^{124}$ I PET/CT a study of remnant radioiodine kinetics comparing the remnant radiation dose in Gy/GBq of the administered ${ }^{131}$ I activity obtained under two stimulation methods: hypothyroidism (THW) versus euthyroidism after the use of rhTSH. The authors retrospectively divided 55 consecutive totally thyroidectomised, radioiodine-naïve patients into two groups. The rhTSH group $(n=16)$ received ${ }^{124} \mathrm{I}$ on continuous thyroid hormone replacement therapy, $24 \mathrm{~h}$ after two consecutive daily i.m. injections of $0.9 \mathrm{mg}$ rhTSH. The THW group $(n=39)$ received ${ }^{124}$ I after weeks-long THW, when serum TSH first measured $\geq 25 \mathrm{mIU} / \mathrm{l}$. PET investigations were performed 4, 24, 48, 72, and $96 \mathrm{~h}$ and PET/CT $25 \mathrm{~h}$ after ${ }^{124} \mathrm{I}$ administration. The mean \pm s.D. specific absorbed dose was statistically equivalent between the groups (rhTSH, $461 \pm 600 \mathrm{~Gy} / \mathrm{GBq} ; \quad$ THW,$\quad 302 \pm$ $329 \mathrm{~Gy} / \mathrm{GBq}$; two-sided $P=0.258$ ). The data presented in these papers suggest that rhTSH or THW delivers statistically comparable radiation doses to thyroid remnant. The authors also note that institutional fixed radioiodine activities formulated for use with THW need not be adjusted for rhTSH-aided ablation.

The small sample size in the rhTSH group might, however, limit the results of the statistical comparison in this work. Further, preferably multicentric clinical trials with higher statistical power are needed for elucidating this finding.

Jentzen et al. (2008), using ${ }^{124} \mathrm{I}$ PET, analyzed iodine kinetics and lesion dose per administered ${ }^{131} \mathrm{I}$ activity of DTC metastases to derive an optimized dosimetry protocol for radioiodine therapy of DTC. The authors evaluated the time-activity-concentration curves of 37 lesions in 17 patients who had undergone near-total thyroidectomy. The lesion dose determination involved ${ }^{124}$ I PET images acquired at 4, 24, 48, 72, and $96 \mathrm{~h}$ after intake of a capsule containing $20-40 \mathrm{MBq}$ of ${ }^{124} \mathrm{I}$. The absorbed doses to the lesions, calculated using data from all five PET time points, served as reference. The lesions were classified into three groups, according to potential for cure with ${ }^{131} \mathrm{I}$ therapy: low ( $\leq 5 \mathrm{~Gy} / \mathrm{GBq} ; n=14$ ), medium (between 5 and $10 \mathrm{~Gy} / \mathrm{GBq} ; n=9)$, or high specific lesion dose ( $>10 \mathrm{~Gy} / \mathrm{GBq} ; n=14$ ). The reference-specific lesion doses were compared with those derived from only 2,3, or 4 PET data points and from one adapted 2-point approach. The results showed that the effective ${ }^{124} \mathrm{I}$ halflife, the specific activity-concentration rate, and the specific $24 \mathrm{~h}$ activity-concentration differed significantly among the groups. The authors conclude that the classification into specific lesion dose groups was feasible using a single PET scan at $\sim 24 \mathrm{~h}$. Because of the highly variable kinetics, one additional 
measurement at $\sim 96 \mathrm{~h}$ was needed to obtain a sufficiently reliable lesion dose estimate. They also state that the adapted 24-96 $\mathrm{h}$ approach appears to be the optimal ${ }^{124}$ I protocol, and is a reliable simplification of the 5-point protocol.

\section{Treatment of childhood cancer}

Thyroid cancer, although very rare in childhood, represents the most common pediatric endocrine neoplasia. The low incidence and the resulting limited availability of prospective, randomized trials lead to a lack of evidence-based recommendations for treatment strategies. Total thyroidectomy and lymphadenectomy, when indicated, followed by ablative radioiodine treatment, are considered the cornerstones of initial patient management which decrease the risk of relapse. On the other hand, less aggressive treatment modalities should also be aimed at due to the high life expectancy of this special patient group and the potential impairment of the quality of life. These considerations have led to individualized 'tailored' therapeutic approaches based on prior risk stratification (Luster et al. 2007).

Hobbs et al. (2009) used a patient-specific 3D radiobiologic dosimetry for ${ }^{131} \mathrm{I}$ treatment planning for an 11-year-old girl with differentiated papillary thyroid cancer with advanced lung involvement, and cerebral metastases. Radioiodine pharmacokinetic assessments and calculation of the recommended administered activity, based on lung toxicity constraints, were performed in real time (i.e. during the data acquisition interval) using ${ }^{124} \mathrm{I}$ PET. The results were made available to the treating physician in time to influence treatment planning, and these estimates were compared with conventional dosimetry methodologies. In subsequent, retrospective analyses, the 3D-absorbed dose calculations were expanded to include additional tumor dose estimates, and the conventional methodologies were reexamined to reveal the causes of the differences observed. A higher recommended administered activity than by the traditionally employed absorbed fraction method (Bolch et al. 2009) with a favorable clinical outcome was obtained. The authors concluded that this approach permitted more aggressive treatment while adhering to patient-specific lung toxicity constraints. Another observation was that a retrospective analysis of the conventional methodologies with appropriate corrections yielded absorbed dose estimates consistent with the 3D-absorbed dose calculation.

\section{Optimized treatment using high activities}

In patients with 'high-risk' DTC, therapy with the highest safe activity appears to be desirable to maximize the tumor radiation dose yet avoid myelotoxicity.

Verburg et al. (2010) sought to evaluate the safety and effectiveness of treating advanced DTC with high ${ }^{131}$ I activities chosen primarily based on the results of dosimetry following the EANM SOP (Lassmann et al. 2008). The authors retrospectively assessed toxicity as well as biochemical and scintigraphic response in their first ten patients receiving such an 'individualized' therapy for advanced DTC. Ten patients received a total of 13 dosimetrically guided treatments with a median administered activity of $14.0 \mathrm{GBq}$ (range: 7.0-21.4 GBq) ${ }^{131}$ I. After 6 of 13 treatment courses in six of ten patients, short-term side effects of ${ }^{131} \mathrm{I}$ therapy, namely nausea, vomiting, or transient sialadenitis, were observed. Leukocyte and platelet counts dropped significantly in the first few weeks after ${ }^{131}$ I treatment, but returned to pretreatment levels by 3 months post-therapy. Serum thyroglobulin levels decreased after 12 of 13 treatments (median reduction: $58 \%$ ) in nine of ten patients. On the basis of their findings, the authors conclude that in their initial patient cohort, high-activity ${ }^{131}$ I therapy for advanced DTC based on pretherapeutic blood dosimetry following the EANM SOP was safe and well tolerated. The authors also note that such treatment almost always produced a partial biochemical tumor response.

Lee et al. (2008) likewise determined the maximal safe activity calculated according to the original Benua approach for 47 patients. Their mean calculated safe activity was $12.5 \mathrm{GBq}$. Twenty-nine patients experienced transient cytopenia after therapy, but three patients did not recover to the baseline level. The authors state that the maximal safe dose provides an effective means of treatment in patients who failed to respond adequately to conventional fixed activity therapy.

Another study on thyroid cancer treatment based on dosimetry is using a slightly different approach. Dorn et al. (2003) reported on the clinical use of dosimetry-guided radioiodine treatment showing the safe and effective application of a 3-Gy bone marrow absorbed dose in 124 patients with DTC who underwent 187 dosimetric evaluations. One of the major shortcomings of this single-center approach, however, is that the dosimetric model used for bone marrow absorbed dose calculations is nonstandard and needs further evaluation. 


\section{The dose to normal organs}

There are three recent studies which assess the absorbed doses to normal organs after radioiodine therapy (Jentzen et al. 2006, 2010, Kolbert et al. 2007), a missing piece in the assessment of DTC patient management.

Kolbert et al. (2007) provide dose volume histograms and mean absorbed doses for 14 normal organs, including organs for which comprehensive doses have not been published (for example, the salivary glands). Calculations were done using a 3D voxel-based method. The highest mean dose, $0.26 \mathrm{~Gy} / \mathrm{GBq}$, was seen in the right submandibular gland, whereas the lowest mean dose, $0.029 \mathrm{~Gy} / \mathrm{GBq}$, was measured in the brain. This study is the first comprehensive approach to normal organ dosimetry in patients by use of a quantitative tomographic imaging modality. An interesting observation is that this work shows good agreement between the absorbed dose to the heart chamber and the blood absorbed dose as measured according to the method of Benua et al. (1962), thus indirectly confirming the validity of the method of Benua et al.

Salivary gland impairment following high activity radioiodine therapy of DTC is a severe side effect with a high impact on patients' quality of life. Dosimetric calculations using planar gamma-camera scintigraphy with ${ }^{131} \mathrm{I}$ and ultrasonography provided evidence that the average organ dose per administered activity is too low to account for observed radiation damages to the salivary glands (Jentzen et al. 2006). In 2010, the authors repeated the study using ${ }^{124}$ I with ten DTC patients who underwent a series of six (or seven) PET scans and one PET/CT scan after administration of $\sim 23 \mathrm{MBq}{ }^{124}$ I iodide (Jentzen et al. 2010). The specific absorbed doses for the submandibular and parotid glands were $0.32 \pm 0.13 \mathrm{~Gy} / \mathrm{GBq}(0.18-0.55)$ and $0.31 \pm 0.10 \mathrm{~Gy} / \mathrm{GBq}(0.13-0.46)$ respectively. No significant differences between ${ }^{124} \mathrm{I}$ PET/CT and ${ }^{131} \mathrm{I}$ dosimetry were found. The authors conclude that a voxel-based calculation taking into account the nonuniform activity distributions in the glands is necessary to possibly explain the radiation-induced salivary gland damage.

\section{Future dosimetry concepts}

The concept of the biological effective dose (BED) is useful to explain the biological effects of radiation, since it is based not only on the absorbed dose, but also on the biological effects such as the competition between the dose rate and the repair rate of sub-lethal single-strand DNA damage. The theoretical basis of this evaluation goes back to the linear-quadratic model, which was introduced in external beam radiation therapy in 1982 (Barendsen 1982) to explain different cellular survival fractions obtained with a fixed absorbed dose but with different dose rates or dose fractionations. One decade ago, Dale developed the theoretical formulas for calculating BED in targeted radionuclide therapy (Dale 1996). The calculation of the BED, which is independent from the irradiation modality, starting from the absorbed dose, allows the comparison of expected biological effects following different irradiation conditions.

Targeted radionuclide therapies are also characterized by strong nonuniformity of dose deposition. Some cells are 'over-killed', while others receive a nonlethal dose. The role of nonuniformity can be accounted for by the equivalent uniform dose (EUD) parameter (O'Donoghue 1999). An EUD of $100 \mathrm{~Gy}$ should give the same effect as a mean dose of $100 \mathrm{~Gy}$ when uniformly distributed.

Prideaux et al. (2007) re-analyzed the pediatric case of diffuse lung micro-metastases, including BED and EUD approach, after the administration of $1.32 \mathrm{GBq}$. The mean absorbed dose, mean biologically effective dose, and EUD for the tumor were 57.7, 58.5, and $25.0 \mathrm{~Gy}$ respectively. Corresponding values for normal lung tissue were 9.5, 9.8, and 8.3 Gy respectively. Their analysis demonstrates the impact of radiobiologic modeling on response prediction. The 57\% reduction in the equivalent dose value for the tumor reflects a high level of dose nonuniformity in the tumor and a corresponding reduced likelihood of achieving a tumor response. Such analyses are expected to be useful in treatment planning for radionuclide therapy.

Another novel approach by Lassmann et al. (2010) is to assess the additional number of DNA double-strand breaks (DSBs) after radioiodine therapy caused by radiation exposure by a dosimetric biomarker. A marker for this DSB formation is the histone $\mathrm{H} 2$ variant $\mathrm{H} 2 \mathrm{AX}(\gamma-\mathrm{H} 2 \mathrm{AX})$. DSBs also attract the damage sensor 53BP1 to the surrounding chromatin, as is the DNA damage sensor 53BP1 that associates with the DSB-surrounding chromatin. The authors studied, in 26 patients after the first ${ }^{131} \mathrm{I}$ therapy, the induction, persistence, and the disappearance of radiation-induced $\gamma-\mathrm{H} 2 \mathrm{AX}$ and 53BP1 foci after the first ${ }^{131} \mathrm{I}$ therapy of patients with differentiated thyroid carcinoma, a model for protracted, continuous, internal whole-body irradiation. Significantly elevated numbers of excess foci/nucleus were still were present 120-144 h after therapy. The number of excess 
radiation-induced foci/nucleus per absorbed dose rate increased with time, potentially indicating a slower rate of DNA repair, or alternatively, a higher de novo rate of focus formation. The authors concluded that radiation-induced $\gamma-\mathrm{H} 2 \mathrm{AX}$ and 53BP1 nuclear foci are useful markers for quantifying DSBs after radioiodine therapy.

\section{Conclusions}

In a majority of patients, the fixed activity-based approach for the treatment of thyroid cancer may have its benefits due to its simplicity. In most cases, this therapy regime stays well below any toxicity limits. The main disadvantage in using this approach is the failure to consider the individuality of the patient. There is evidence for over- or more probably undertreatment of individual patients without realizing it. In addition, the efficacy of several treatments in one patient might be degraded.

In the case of lesion-based dosimetry, the pretherapeutic use of ${ }^{124}$ I seems to be state of the art, although the matter of stunning has not been settled. In addition, careful calibration of the PET/CT scanners is mandatory. In the lesion-based approach, the pretherapeutic or therapeutic administration of ${ }^{131}$ I does not take into account regional differences in the local iodine uptake.

Patient-specific blood-based dosimetry is comparatively easy to perform before and during therapy. In selected patient cases, this procedure will allow extending the activity beyond the limit of therapies using fixed activities and will reduce the risk of severe side effects.

\section{Declaration of interest}

The authors declare that there is no conflict of interest that could be perceived as prejudicing the impartiality of the research reported.

\section{Funding}

This research did not receive any specific grant from any funding agency in the public, commercial, or not-for-profit sector.

\section{References}

Barendsen GW 1982 Dose fractionation, dose rate and iso-effect relationships for normal tissue responses. International Journal of Radiation Oncology, Biology, Physics 8 1981-1997.
Benua RS, Cicale NR, Sonenberg M \& Rawson RW 1962 The relation of radioiodine dosimetry to results and complications in the treatment of metastatic thyroid cancer. American Journal of Roentgenology, Radium Therapy, and Nuclear Medicine 87 171-182.

Bolch WE, Eckerman KF, Sgouros G \& Thomas SR 2009 MIRD pamphlet No. 21: a generalized schema for radiopharmaceutical dosimetry - standardization of nomenclature. Journal of Nuclear Medicine $\mathbf{5 0}$ 477-484.

Capoccetti F, Criscuoli B, Rossi G, Ferretti F, Manni C \& Brianzoni E 2009 The effectiveness of ${ }^{124}$ I PET/CT in patients with differentiated thyroid cancer. Quarterly Journal of Nuclear Medicine and Molecular Imaging 53 536-545.

Chiesa C, Castellani MR, Vellani C, Orunesu E, Negri A, Azzeroni R, Botta F, Maccauro M, Aliberti G, Seregni E et al. 2009 Individualized dosimetry in the management of metastatic differentiated thyroid cancer. Quarterly Journal of Nuclear Medicine and Molecular Imaging 53 546-561.

Crawford DC, Flower MA, Pratt BE, Hill C, Zweit J, McCready VR \& Harmer CL 1997 Thyroid volume measurement in thyrotoxic patients: comparison between ultrasonography and iodine-124 positron emission tomography. European Journal of Nuclear Medicine $\mathbf{2 4}$ 1470-1478.

Dale RG 1996 Dose-rate effects in targeted radiotherapy. Physics in Medicine and Biology 41 1871-1884.

De Klerk JM \& Oyen WJ 2009 The continuous debate in literature about the usage of iodine-131 dosing for the ablation of thyroid remnants and metastases. Minerva Endocrinologica 34 57-69.

Dorn R, Kopp J, Vogt H, Heidenreich P, Carroll RG \& Gulec SA 2003 Dosimetry-guided radioactive iodine treatment in patients with metastatic differentiated thyroid cancer: largest safe dose using a riskadapted approach. Journal of Nuclear Medicine 44 451-456.

Eschmann SM, Reischl G, Bilger K, Kupferschlager J, Thelen MH, Dohmen BM, Besenfelder H \& Bares R 2002 Evaluation of dosimetry of radioiodine therapy in benign and malignant thyroid disorders by means of iodine-124 and PET. European Journal of Nuclear Medicine and Molecular Imaging 29 760-767.

Flux GD, Haq M, Chittenden SJ, Buckley S, Hindorf C, Newbold K \& Harmer CL 2010 A dose-effect correlation for radioiodine ablation in differentiated thyroid cancer. European Journal of Nuclear Medicine and Molecular Imaging 37 270-275.

Freudenberg LS, Jentzen W, Gorges R, Petrich T, Marlowe RJ, Knust J \& Bockisch A $2007{ }^{124}$ I-PET dosimetry in advanced differentiated thyroid cancer: therapeutic impact. Nuklearmedizin 46 121-128. 
Freudenberg LS, Fromke C, Petrich T, Marlowe RJ, Koska WW, Brandau W, Eising EG, Knust EJ, Bockisch A \& Jentzen W 2009 Thyroid remnant dose: ${ }^{124}$ I-PET/CT dosimetric comparison of rhTSH versus thyroid hormone withholding before radioiodine remnant ablation in differentiated thyroid cancer. Experimental and Clinical Endocrinology and Diabetes [in press].

Hänscheid H, Lassmann M, Luster M, Thomas SR, Pacini F, Ceccarelli C, Ladenson PW, Wahl RL, Schlumberger M, Ricard M et al. 2006 Iodine biokinetics and dosimetry in radioiodine therapy of thyroid cancer: procedures and results of a prospective international controlled study of ablation after rhTSH or hormone withdrawal. Journal of Nuclear Medicine 47 648-654.

Hänscheid H, Lassmann M, Luster M, Kloos RT \& Reiners C 2009 Blood dosimetry from a single measurement of the whole body radioiodine retention in patients with differentiated thyroid carcinoma. Endocrine-Related Cancer 16 1283-1289.

Haq MS, McCready RV \& Harmer CL 2004 Treatment of advanced differentiated thyroid carcinoma with high activity radioiodine therapy. Nuclear Medicine Communications 25 799-805.

Hobbs RF, Wahl RL, Lodge MA, Javadi MS, Cho SY, Chien DT, Ewertz ME, Esaias CE, Ladenson PW \& Sgouros G $2009{ }^{124}$ I PET-based 3D-RD dosimetry for a pediatric thyroid cancer patient: real-time treatment planning and methodologic comparison. Journal of Nuclear Medicine 50 1844-1847.

Jentzen W, Schneider E, Freudenberg L, Eising EG, Gorges R, Muller SP, Brandau W \& Bockisch A 2006 Relationship between cumulative radiation dose and salivary gland uptake associated with radioiodine therapy of thyroid cancer. Nuclear Medicine Communications 27 669-676.

Jentzen W, Freudenberg L, Eising EG, Sonnenschein W, Knust J \& Bockisch A 2008 Optimized ${ }^{124}$ I PET dosimetry protocol for radioiodine therapy of differentiated thyroid cancer. Journal of Nuclear Medicine 49 1017-1023.

Jentzen W, Hobbs RF, Stahl A, Knust J, Sgouros G \& Bockisch A 2010 Pre-therapeutic ${ }^{124}$ I PET(/CT) dosimetry confirms low average absorbed doses per administered ${ }^{131} \mathrm{I}$ activity to the salivary glands in radioiodine therapy of differentiated thyroid cancer. European Journal of Nuclear Medicine and Molecular Imaging 37 884-895.

de Keizer B, Brans B, Hoekstra A, Zelissen PM, Koppeschaar HP, Lips CJ, van Rijk PP, Dierckx RA \& de Klerk JM 2003 Tumour dosimetry and response in patients with metastatic differentiated thyroid cancer using recombinant human thyrotropin before radioiodine therapy. European Journal of Nuclear Medicine and Molecular Imaging 30 367-373.

Kolbert KS, Pentlow KS, Pearson JR, Sheikh A, Finn RD, Humm JL \& Larson SM 2007 Prediction of absorbed dose to normal organs in thyroid cancer patients treated with ${ }^{131}$ I by use of ${ }^{124}$ I PET and 3-dimensional internal dosimetry software. Journal of Nuclear Medicine 48 143-149.

Kulkarni K, Van Nostrand D, Atkins F, Aiken M, Burman K \& Wartofsky L 2006 The relative frequency in which empiric dosages of radioiodine would potentially overtreat or undertreat patients who have metastatic welldifferentiated thyroid cancer. Thyroid 16 1019-1023.

Lassmann M, Luster M, Hänscheid H \& Reiners C 2004 Impact of ${ }^{131}$ I diagnostic activities on the biokinetics of thyroid remnants. Journal of Nuclear Medicine $\mathbf{4 5}$ 619-625.

Lassmann M, Hanscheid H, Chiesa C, Luster M, Bombardieri E \& Reiners C 2007 Blood dose calculation in radioiodine therapy of differentiated thyroid cancer. European Journal of Nuclear Medicine and Molecular Imaging 34 S199.

Lassmann M, Hänscheid H, Chiesa C, Hindorf C, Flux G \& Luster M 2008 EANM Dosimetry Committee series on standard operational procedures for pre-therapeutic dosimetry I: blood and bone marrow dosimetry in differentiated thyroid cancer therapy. European Journal of Nuclear Medicine and Molecular Imaging 35 1405-1412.

Lassmann M, Hänscheid H, Gassen D, Biko J, Meineke V, Reiners C \& Scherthan H 2010 In-vivo $\gamma$-H2AX and 53BP1 focus formation in blood cells after radioiodine therapy of differentiated thyroid cancer. Journal of Nuclear Medicine [in press].

Lee JJ, Chung JK, Kim SE, Kang WJ, Park do J, Lee DS, Cho BY \& Lee MC 2008 Maximal safe dose of I-131 after failure of standard fixed dose therapy in patients with differentiated thyroid carcinoma. Annals of Nuclear Medicine 22 727-734.

Leeper RD 1985 Thyroid cancer. Medical Clinics of North America 69 1079-1096.

Li WB, Friedland W, Pomplun E, Jacob P, Paretzke HG, Lassmann M \& Reiners C 2001 Track structures and dose distributions from decays of I-131 and I-125 in and around water spheres simulating micrometastases of differentiated thyroid cancer. Radiation Research 156 419-429.

Luster M, Sherman SI, Skarulis MC, Reynolds JR, Lassmann M, Hänscheid H \& Reiners C 2003 Comparison of radioiodine biokinetics following the administration of recombinant human thyroid stimulating hormone and after thyroid hormone withdrawal in thyroid carcinoma. European Journal of Nuclear Medicine and Molecular Imaging 30 1371-1377.

Luster M, Lassmann M, Freudenberg LS \& Reiners C 2007 Thyroid cancer in childhood: management strategy, including dosimetry and long-term results. Hormones 6 269-278.

Luster M, Clarke SE, Dietlein M, Lassmann M, Lind P, Oyen WJ, Tennvall J \& Bombardieri E 2008 Guidelines for 
radioiodine therapy of differentiated thyroid cancer. European Journal of Nuclear Medicine and Molecular Imaging 35 1941-1959.

Maxon HR III \& Smith HS 1990 Radioiodine-131 in the diagnosis and treatment of metastatic well differentiated thyroid cancer. Endocrinology and Metabolism Clinics of North America 19 685-718.

Maxon HR, Thomas SR, Hertzberg VS, Kereiakes JG, Chen IW, Sperling MI \& Saenger EL 1983 Relation between effective radiation dose and outcome of radioiodine therapy for thyroid cancer. New England Journal of Medicine 309 937-941.

Medvedec M 2005 Thyroid stunning in vivo and in vitro. Nuclear Medicine Communications 26 731-735.

Meier DA, Brill DR, Becker DV, Clarke SE, Silberstein EB, Royal HD \& Balon HR 2002 Procedure guideline for therapy of thyroid disease with (131)iodine. Journal of Nuclear Medicine 43 856-861.

Menzel C, Grunwald F, Schomburg A, Palmedo H, Bender H, Spath G \& Biersack HJ 1996 "High-dose" radioiodine therapy in advanced differentiated thyroid carcinoma. Journal of Nuclear Medicine 37 1496-1503.

O'Donoghue JA 1999 Implications of nonuniform tumor doses for radioimmunotherapy. Journal of Nuclear Medicine 40 1337-1341.

Pacini F, Ladenson PW, Schlumberger M, Driedger A, Luster M, Kloos RT, Sherman S, Haugen B, Corone C, Molinaro E et al. 2006 Radioiodine ablation of thyroid remnants after preparation with recombinant human thyrotropin in differentiated thyroid carcinoma: results of an international, randomized, controlled study. Journal of Clinical Endocrinology and Metabolism 91 926-932.

Pentlow KS, Graham MC, Lambrecht RM, Daghighian F, Bacharach SL, Bendriem B, Finn RD, Jordan K, Kalaigian H, Karp JS et al. 1996 Quantitative imaging of iodine-124 with PET. Journal of Nuclear Medicine 37 1557-1562.

Prideaux AR, Song H, Hobbs RF, He B, Frey EC, Ladenson PW, Wahl RL \& Sgouros G 2007 Three-dimensional radiobiologic dosimetry: application of radiobiologic modeling to patient-specific 3-dimensional imagingbased internal dosimetry. Journal of Nuclear Medicine $\mathbf{4 8}$ 1008-1016.

Remy H, Borget I, Leboulleux S, Guilabert N, Lavielle F, Garsi J, Bournaud C, Gupta S, Schlumberger M \& Ricard M $2008{ }^{131}$ I effective half-life and dosimetry in thyroid cancer patients. Journal of Nuclear Medicine $\mathbf{4 9}$ $1445-1450$.

Salvatori M \& Luster M 2010 Radioiodine therapy dosimetry in benign thyroid disease and differentiated thyroid carcinoma. European Journal of Nuclear Medicine and Molecular Imaging 37 821-828.
Samuel AM, Rajashekharrao B \& Shah DH 1998 Pulmonary metastases in children and adolescents with welldifferentiated thyroid cancer. Journal of Nuclear Medicine 39 1531-1536.

Schlumberger M \& Pacini F 2003 Thyroid Tumors. Paris: Editions Nucléon.

Sgouros G, Kolbert KS, Sheikh A, Pentlow KS, Mun EF, Barth A, Robbins RJ \& Larson SM 2004 Patientspecific dosimetry for ${ }^{131} \mathrm{I}$ thyroid cancer therapy using ${ }^{124}$ I PET and 3-dimensional-internal dosimetry (3D-ID) software. Journal of Nuclear Medicine 45 1366-1372.

Sgouros G, Song H, Ladenson PW \& Wahl RL 2006 Lung toxicity in radioiodine therapy of thyroid carcinoma: development of a dose-rate method and dosimetric implications of the 80-mCi rule. Journal of Nuclear Medicine 47 1977-1984.

Siegel JA, Thomas SR, Stubbs JB, Stabin MG, Hays MT, Koral KF, Robertson JS, Howell RW, Wessels BW, Fisher DR et al. 1999 MIRD pamphlet no. 16: techniques for quantitative radiopharmaceutical biodistribution data acquisition and analysis for use in human radiation dose estimates. Journal of Nuclear Medicine 40 37S-61S.

Song H, He B, Prideaux A, Du Y, Frey E, Kasecamp W, Ladenson PW, Wahl RL \& Sgouros G 2006 Lung dosimetry for radioiodine treatment planning in the case of diffuse lung metastases. Journal of Nuclear Medicine 47 1985-1994.

Stabin MG, Sparks RB \& Crowe E 2005 OLINDA/EXM: the second-generation personal computer software for internal dose assessment in nuclear medicine. Journal of Nuclear Medicine 46 1023-1027.

Thomas SR, Samaratunga RC, Sperling M \& Maxon HR III 1993 Predictive estimate of blood dose from external counting data preceding radioiodine therapy for thyroid cancer. Nuclear Medicine and Biology 20 157-162.

Tuttle RM, Leboeuf R, Robbins RJ, Qualey R, Pentlow K, Larson SM \& Chan CY 2006 Empiric radioactive iodine dosing regimens frequently exceed maximum tolerated activity levels in elderly patients with thyroid cancer. Journal of Nuclear Medicine $\mathbf{4 7}$ 1587-1591.

Van Nostrand D, Atkins F, Yeganeh F, Acio E, Bursaw R \& Wartofsky L 2002 Dosimetrically determined doses of radioiodine for the treatment of metastatic thyroid carcinoma. Thyroid 12 121-134.

Verburg FA, Hanscheid H, Biko J, Hategan MC, Lassmann M, Kreissl MC, Reiners C \& Luster M 2010 Dosimetryguided high-activity (131)I therapy in patients with advanced differentiated thyroid carcinoma: initial experience. European Journal of Nuclear Medicine and Molecular Imaging 37 896-903. 